\title{
Adaptive Backstepping Control for a Fully-Actuated Rigid-Body in a Dual-Quaternion Framework*
}

\author{
Tom Stian Andersen ${ }^{1}$ and Raymond Kristiansen ${ }^{2}$
}

\begin{abstract}
We address the trajectory tracking problem for a fully actuated rigid-body with unknown mass and inertia parameters and unknown disturbance forces, using an adaptive backstepping controller based on dual-quaternions. We show that the proposed controller, in closed loop with a nonlinear model of the system, renders the equilibrium points uniformly asymptotically stable. The proposed controller is proved to be uniformely asymptotically stable. Numerical simulations are provided to demonstrate the performance of the controller. In addition it is shown through a numeric example that the parameter update law for the mass and inertia parameters can converge to the true mass and inertia parameters with a proper choice of desired trajectory.
\end{abstract}

\section{INTRODUCTION}

Motion control of rigid-bodies is a fundamental problem within control theory, since many physical systems such as satellites, aircrafts, land vehicles and ships can be idealized as rigid-bodies. The representation of attitude motion of rigid-bodies has been extensively researched, because of the many possible representations such as direction cosine matrices, Euler angles and quaternions. In recent years a representation of rigid-body motion using unit dual-quaternions has been considered. This representation combines translation and rotation into a unified framework and allows for efficient and compact notation. Moreover by combining translation and rotational motion in a single framework the total motion of the rigid-body can be controlled with a single controller.

A considerable amount of research has been done on the use of dual-quaternions in theoretical kinematics. The original motivation is that the motion of a rigid-body in three-dimensional Euclidian space can be described by six parameters, which can be regarded as a point in a sixdimensional space. Work was carried out by Study in [1] to apply the work of Clifford [2] to the kinematics of rigidbodies where the motion of a rigid-body can be seen as a point on a six-dimensional manifold in eight-dimensional space. This idea was further developed by [3] to represent Euclidian displacements using four coordinates in a dualspace to study the kinematic motion of rigid-bodies.

In [4] dual-numbers were used to express the sixdimensional motion of a rigid-body in a three-dimensional

\footnotetext{
*This research has been funded by the Norwegian Research Council and is part of the Sikrere logistikk fra farty med ubemannet logistikkhelikopter project 282317.

${ }^{1}$ Tom Stian Andersen is with the Department of Electrical Engineering, UiT The Arctic University of Norway, 8515 Narvik, Norway tom.s.andersen@uit.no

${ }^{2}$ Raymond Kristiansen is with the Department of Electrical Engineering, UiT The Arctic University of Norway, 8515 Narvik, Norway raymond.kristiansen@uit.no
}

dual-space by the use of a dual inertia operator. This work was later used in [5] to study the control of satellite formations using a PD-like tracking controller based on the logarithm of dual-quaternions. The work of [3] was later used by [6] to solve the general dynamics problem using dual-quaternions. Dual-quaternions has also been applied in the study of navigation in [7] where navigational equations of motion are derived using dual-quaternions and the resulting algorithms were shown to be suitable for high precision navigation systems. Although the study of dual-quaternions is prevalent in kinematics and the use of dual-numbers in rigid-body dynamics, less work has been done on the application of dual-quaternions on the dynamics of rigidbodies.

Most of the applications of dual-quaternions has been centered around satellite pose control [5], [8], [9], [10]. A notable exception is in [11] where a dual-quaternion controller is derived to solve an underactuated trajectory tracking problem. In [9] the set-point regulation problem is addressed and a linear and angular velocity-free controller is derived. In [12] a velocity-free tracking controller is proposed with a dual-quaternion filter that provides dampening injection while in [13] a discontinuous backstepping controller is derived that solves the manoeuver problem for a rigid spacecraft. In [14] an adaptive control law is developed for tracking which takes into account unknown mass and inertia as well as unknown disturbances. In [15] an adaptive control law is developed for satellite proximity operations between a target satellite and a chaser satellite which does not depend on knowing the mass and inertia parameters of the chaser satellite. In addition the controller also compensates for constant or slowly time-varying disturbances. In [16] an adaptive controller that estimates the mass and inertia parameters using concurrent learning is derived. The proposed adaptive controller was based on concurrent learning methods which avoid the need for a persistently exciting reference trajectory as needed in [15].

In this paper a dual-quaternion adaptive backstepping tracking controller is introduced for a fully-actuated rigidbody which can easily be adapted to specific problems such as satellite pose tracking control. In contrast with earlier works [15], [14] and [16], the dual-inertia matrix is kept anti-diagonal such that it is consistent with the modelling framework of [4]. Furthermore it avoids the use of the swap operator, leading to a simpler controller design. A numerical simulation is presented which shows that the controller can indentify the mass and inertia parameters if the desired trajectory is chosen in accordance with [17]. 
The outline of this paper is as follows. Section II presents the essential preliminaries associated with dual-quaternions. Section III introduces the kinematics and dynamics of rigidbodies using dual-quaternions. Section IV outlines the problem statement, error kinematics, control design and stability analysis. Section $\mathrm{V}$ provides a numerical simulation results and a conclusion is provided in Section VI.

\section{PRELIMINARIES}

\section{A. Notation and Reference frames}

Vectors are denoted as lower-case bold letters while scalars are non-bold for instance $\boldsymbol{x} \in \mathbb{R}^{n}$ is an n-dimensional vector while $a \in \mathbb{R}$ is a scalar. Matrices are upper-case bold letters where the transpose of an $n \times m$ matrix $M \in \mathbb{R}^{n \times m}$ is denoted $\boldsymbol{M}^{\top}$. The $n \times n$ identity matrix is denoted $\boldsymbol{I}_{n \times n}$ while an $n \times m$ matrix with zero entries is denoted $\mathbf{0}_{n \times m}$. The matrix norm is defined as $\|\boldsymbol{A}\|=\max \{\sqrt{\lambda}$ : $\lambda$ is an eigenvalue of $\left.\boldsymbol{A}^{\top} \boldsymbol{A}\right\}$. The skew operator $S(\cdot)$ is defined as

$$
S(\boldsymbol{x})=\left[\begin{array}{ccc}
0 & -x_{3} & x_{2} \\
x_{3} & 0 & -x_{1} \\
-x_{2} & x_{1} & 0
\end{array}\right], \quad \boldsymbol{x} \in \mathbb{R}^{3}
$$

with $\boldsymbol{x}=\left[\begin{array}{lll}x_{1} & x_{2} & x_{3}\end{array}\right]^{\top}$ such that for two vectors $\boldsymbol{a}, \boldsymbol{b} \in$ $\mathbb{R}^{3}, S(\boldsymbol{a}) \boldsymbol{b}=\boldsymbol{a} \times \boldsymbol{b}$. The $D(\cdot)$ operator is defined as

$$
D(\boldsymbol{x})=\left[\begin{array}{ccc}
x_{2} & x_{3} & 0 \\
x_{1} & 0 & x_{3} \\
0 & x_{1} & x_{2}
\end{array}\right], \quad \boldsymbol{x} \in \mathbb{R}^{3} .
$$

The derivative with respect to time is denoted as $\dot{\boldsymbol{x}}=\frac{d \boldsymbol{x}}{d t}$. Reference frames are denoted $\mathcal{F}_{(\cdot)}$, and superscripts are used to denote a vector frame of reference, such that the vector $\boldsymbol{x}^{A}$ is expressed in $\mathcal{F}_{A}$. Angular velocities are denoted as $\boldsymbol{\omega}_{A, B}^{C} \in \mathbb{R}^{3}$ which is the angular velocity of $\mathcal{F}_{B}$ relative to $\mathcal{F}_{A}$, expressed in $\mathcal{F}_{C}$. The vector norm is the Euclidean norm denoted as $\|\boldsymbol{x}\|=\langle\boldsymbol{x}, \boldsymbol{x}\rangle^{\frac{1}{2}}$. The set of quaternions is defined as

$$
\mathbb{H}:=\left\{\left(q_{0}, \boldsymbol{q}_{v}\right): q_{0} \in \mathbb{R}, \boldsymbol{q}_{v} \in \mathbb{R}^{3}\right\}
$$

where $q_{0}$ is the scalar part and $\boldsymbol{q}_{v}$ is the vector part, while the set of unit-quaternions is defined as $\mathbb{H}_{u}:=$ $\{\boldsymbol{q} \in \mathbb{H}:\|\boldsymbol{q}\|=1\}$. The set of vector quaternions is defined as $\mathbb{H}_{v}:=\left\{\boldsymbol{q} \in \mathbb{H}: q_{0}=0\right\}$.

The reference frames that are used in this work are given as

Inertial frame This reference frame denoted $\mathcal{F}_{i}$ has its origin at a fixed point in space and its axes are fixed.

Body frame This coordinate reference frame denoted $\mathcal{F}_{b}$ is fixed at the rigid-body's centre of mass and the axes are fixed to the rigid-body.

Desired frame This coordinate reference frame denoted $\mathcal{F}_{d}$ represents the rigid-body's desired pose.

\section{B. Dual quaternions}

Unit dual-quaternions are defined as [3]

$\hat{\mathbb{H}}_{u}:=\left\{\hat{q}=\boldsymbol{q}_{p}+\epsilon \boldsymbol{q}_{d}: \boldsymbol{q}_{p} \in \mathbb{H}_{u}, \boldsymbol{q}_{p} \otimes \boldsymbol{q}_{d}^{*}+\boldsymbol{q}_{d} \otimes \boldsymbol{q}_{p}^{*}=\mathbf{0}\right\}$

where $\epsilon$ is the dual unit with the property $\epsilon^{2}=0, \epsilon \neq 0, \boldsymbol{q}_{p}$ is called the primary part and $\boldsymbol{q}_{d}$ is called the dual part. The set of dual-vectors is defined as

$$
\hat{\mathbb{H}}_{v}:=\left\{\hat{q}=\boldsymbol{q}_{p}+\epsilon \boldsymbol{q}_{d}: \boldsymbol{q}_{p} \in \mathbb{H}_{v}, \boldsymbol{q}_{d} \in \mathbb{H}_{v}\right\} .
$$

The quaternion product between two unit dual-quaternions $\hat{q}_{1}$ and $\hat{q}_{2}$ is defined as

$$
\hat{q}_{1} \otimes \hat{q}_{2}=\boldsymbol{q}_{1, p} \otimes \boldsymbol{q}_{2, p}+\epsilon\left(\boldsymbol{q}_{1, p} \otimes \boldsymbol{q}_{2, d}+\boldsymbol{q}_{1, d} \otimes \boldsymbol{q}_{2, p}\right)
$$

where $\boldsymbol{q}_{1, p} \otimes \boldsymbol{q}_{2, p}$ denotes the quaternion product. Addition and subtraction of two dual-quaternions is defined as

$$
\hat{q}_{1} \pm \hat{q}_{2}=\boldsymbol{q}_{1, p} \pm \boldsymbol{q}_{2, p}+\epsilon\left(\boldsymbol{q}_{1, d} \pm \boldsymbol{q}_{2, d}\right) .
$$

The dual-quaternion conjugate is defined as $\hat{q}^{*}=\boldsymbol{q}_{p}^{*}+\epsilon \boldsymbol{q}_{d}^{*}$. In this paper the inner product between two dual-quaternions is defined as

$$
\left\langle\hat{q}_{1}, \hat{q}_{2}\right\rangle=\left\langle\boldsymbol{q}_{1, p}, \boldsymbol{q}_{2, p}\right\rangle+\left\langle\boldsymbol{q}_{1, d}, \boldsymbol{q}_{2, d}\right\rangle
$$

which defines the norm of a dual-quaternion as

$$
\|\hat{q}\|=\langle\hat{q}, \hat{q}\rangle^{\frac{1}{2}}=\sqrt{\left(\boldsymbol{q}_{p}\right)^{\top} \boldsymbol{q}_{p}+\left(\boldsymbol{q}_{d}\right)^{\top} \boldsymbol{q}_{d}} .
$$

The identity unit dual-quaternion is defined as $\hat{q}_{I}=\boldsymbol{q}_{I}+\epsilon \mathbf{0}$ and $\hat{0}=\mathbf{0}+\epsilon \mathbf{0}$ denotes the zero element for dual-vectors.

\section{DUAL-QUATERniOn Rigid-BODY KinEMATICS AND DYNAMICS}

\section{A. Kinematics}

The position and orientation of a rigid-body relative to some inertial reference frame $\mathcal{F}_{i}$ can be compactly expressed through a dual-quaternion [18]

$$
\hat{q}=\boldsymbol{q}_{i, b}+\epsilon \frac{1}{2} \boldsymbol{p}^{i} \otimes \boldsymbol{q}_{i, b}=\boldsymbol{q}_{i, b}+\epsilon \frac{1}{2} \boldsymbol{q}_{i, b} \otimes \boldsymbol{p}^{b}
$$

where $\boldsymbol{p}^{i} \in \mathbb{H}_{v}$ is the rigid-body's inertial position while $\boldsymbol{q}_{i, b} \in \mathbb{H}_{u}$ represents the rigid-body's attitude. As the rigidbody moves and rotates the dual-quaternion will change over time, according to

$$
\dot{\hat{q}}=\frac{1}{2} \hat{q} \otimes \hat{\omega}^{b}
$$

where $\hat{\omega}^{b}=\boldsymbol{\omega}_{i, b}^{b}+\epsilon \boldsymbol{v}^{b}$ is called the dual velocity.

\section{B. Dynamics}

It was shown in [19] that the dual velocity can be related to the dual momentum through a dual inertia operator

$$
\hat{h}^{b}=\hat{M} \hat{\omega}^{b}
$$

where $\hat{h}^{b}=\boldsymbol{h}_{L}+\epsilon \boldsymbol{h}_{A}$ with $\boldsymbol{h}_{L}$ representing the linear momentum and $\boldsymbol{h}_{A}$ representing the angular momentum. The dual inertia operator is a matrix with dual number elements, 
however it has also been shown that $\hat{M}$ can be defined as [18]

$$
\hat{M}=\left[\begin{array}{cccc}
0 & \mathbf{0}_{1,3} & 1 & \mathbf{0}_{1,3} \\
\mathbf{0}_{3,1} & \mathbf{0}_{3 x 3} & \mathbf{0}_{3,1} & m \boldsymbol{I}_{3} \\
1 & \mathbf{0}_{1,3} & 0 & \mathbf{0}_{1,3} \\
\mathbf{0}_{3,1} & \boldsymbol{J}^{b} & \mathbf{0}_{1 x 3} & \mathbf{0}_{3,3}
\end{array}\right] .
$$

where $m \in \mathbb{R}$ is the mass of the rigid-body while $\boldsymbol{J}^{b} \in$ $\mathbb{R}^{3 \times 3}$ denotes the inertia matrix. The dual inertia operator in the form (7) always has an inverse and the product with its inverse yields the identity matrix as shown in [18]. The dual force is related to the derivative of the dual momentum which is expressed as

$$
\hat{M} \dot{\hat{\omega}}^{b}=\hat{f}_{u}^{b}-\hat{f}_{d}^{b}-\hat{f}_{G}^{b}-\hat{\omega}^{b} \times \hat{M} \hat{\omega}^{b}
$$

where $\hat{f}_{G}^{b}=\boldsymbol{f}_{G}^{b}+\epsilon \mathbf{0}$ is the gravitational forces expressed in the body frame, $\hat{f}_{d}^{b}$ represents a constant and bounded unknown disturbance dual-force while $\hat{f}_{u}^{b}=\boldsymbol{f}^{b}+\epsilon \boldsymbol{\tau}^{b}$ with $f^{b} \in \mathbb{H}_{v}$ and $\tau^{b} \in \mathbb{H}_{v}$ represents the combined applied forces and moments in the body frame and in this work it specifically represents the control force to be designed.

\section{Controller DESigN}

\section{A. Problem statement}

Consider a fully actuated rigid-body with kinematics and dynamics described by (5) and (7) respectively. Let a desired dual-quaternion be defined as $\hat{q}_{d}(t)=\boldsymbol{q}_{i, d}+\epsilon \frac{1}{2} \boldsymbol{q}_{i, d} \otimes \boldsymbol{p}^{d}$ and let it be a known two times continuously differentiable bounded time-varying trajectory, i.e. $\hat{q}_{d}, \hat{\omega}_{d}^{d}, \dot{\hat{\omega}}_{d}^{d} \in \mathcal{L}_{\infty}$. Design a feedback law that ensures that $\hat{q} \rightarrow \hat{q}_{d}$ and $\hat{\omega} \rightarrow \hat{\omega}_{d}$ as $t \rightarrow \infty$.

\section{B. Error kinematics}

Let the error dual-quaternion be defined as

$$
\begin{aligned}
\hat{q}_{e} & =\hat{q}_{d}^{*} \otimes \hat{q} \\
& =\boldsymbol{q}_{e}+\epsilon \frac{1}{2} \boldsymbol{q}_{e} \otimes \boldsymbol{p}_{e}^{b}
\end{aligned}
$$

where $\boldsymbol{q}_{e}=\boldsymbol{q}_{d, b}=\boldsymbol{q}_{i, d}^{*} \otimes \boldsymbol{q}_{i, b}$ is the orientation error and $\boldsymbol{p}_{e}^{b}=\boldsymbol{p}^{b}-\boldsymbol{p}_{d}^{b}$ is the position error expressed in the body frame. The derivative of (8) is

$$
\begin{aligned}
& \dot{\hat{q}}_{e}=\frac{1}{2} \hat{q}_{e} \otimes \hat{\omega}_{e}^{b} \\
& \hat{\omega}_{e}^{b}=\hat{\omega}^{b}-\hat{q}_{e}^{*} \otimes \hat{\omega}_{d}^{d} \otimes \hat{q}_{e}=\boldsymbol{\omega}_{e}^{b}+\epsilon \boldsymbol{v}_{e}^{b}-\boldsymbol{\omega}_{i, d}^{b} \times \boldsymbol{p}_{e}^{b}
\end{aligned}
$$

where $\hat{\omega}_{d}^{d}=\boldsymbol{\omega}_{i, d}^{d}+\epsilon \boldsymbol{v}_{d}^{d}$ is the desired dual velocity, $\boldsymbol{\omega}_{e}^{b}=$ $\boldsymbol{\omega}_{i, b}^{b}-\boldsymbol{\omega}_{i, d}^{b}$ is the angular velocity error expressed in the body frame and $\boldsymbol{v}_{e}^{b}=\boldsymbol{v}^{b}-\boldsymbol{v}_{d}^{b}$ is the velocity error expressed in the body frame.

\section{Integrator backstepping}

In [20] an integrator backstepping control law is derived under the following assumptions:

a) Assumption 1: $q_{e, 0}(t) q_{e, 0}\left(t_{0}\right) \geq 0, \forall t>t_{0}$ were $q_{e, 0}$ is the scalar part of $\boldsymbol{q}_{e}$.

b) Assumption 2: The mass $m$ and inertia matrix $\boldsymbol{J}^{b}$ are constant and known. In addition $\boldsymbol{J}^{b}$ is a symmetric and positive definite matrix. c) Assumption 3: $\hat{q}_{d}, \hat{\omega}_{d}^{d}, \dot{\hat{\omega}}_{d}^{d} \in \mathcal{L}_{\infty}$ and $\hat{q}_{d}, \hat{\omega}_{d}^{d}, \dot{\hat{\omega}}_{d}^{d}$ are assumed to be known.

Under Assumption 1-3 the control law in [20] can be written as

$$
\hat{f}_{u}^{b}=\hat{f}_{G}^{b}+\hat{f}_{d}^{b}+\hat{\omega}^{b} \times \hat{M} \hat{\omega}^{b}+\hat{M} \dot{\hat{\omega}}_{c}^{b}-\hat{K}\left(\hat{K}_{2} \hat{z}+\hat{\zeta}\right)
$$

where $\hat{K}$ is an anti-diagonal matrix such that $\hat{K} \hat{M}$ is symmetric and positive definite, and $\hat{K}_{2}$ is a positive definite matrix of the form

$$
\hat{K}_{2}=\left[\begin{array}{cccc}
0 & \mathbf{0}_{1,3} & 0 & \mathbf{0}_{1,3} \\
\mathbf{0}_{3,1} & k_{\omega} \boldsymbol{I}_{3 \times 3} & \mathbf{0}_{3,1} & \mathbf{0}_{3 x 3} \\
0 & \mathbf{0}_{1,3} & 0 & \mathbf{0}_{1,3} \\
\mathbf{0}_{3,1} & \mathbf{0}_{3,3} & \mathbf{0}_{1 \times 3} & k_{v} \boldsymbol{I}_{3 \times 3}
\end{array}\right] .
$$

with $k_{\omega}>0$ and $k_{v}>0$. In (10) the term $\hat{\zeta}=\boldsymbol{q}_{e, v}+\epsilon \frac{1}{2} \boldsymbol{p}_{e}^{b}$ represents the orientation and position error. Let the error variable $\hat{z}$ be defined as

$$
\hat{z}=\hat{\omega}^{b}-\hat{\omega}_{c}^{b}
$$

where $\hat{\omega}_{c}^{b}$ is the desired value for the virtual control input $\hat{\omega}^{b}$ and is defined as

$$
\hat{\omega}_{c}^{b}=\hat{q}_{e}^{*} \otimes \hat{\omega}_{d}^{d} \otimes \hat{q}_{e}-\hat{K}_{1} \hat{\zeta}
$$

where $\hat{K}_{1}$ is a positive definite matrix of the form

$$
\hat{K}_{1}=\left[\begin{array}{cccc}
0 & \mathbf{0}_{1,3} & 0 & \mathbf{0}_{1,3} \\
\mathbf{0}_{3,1} & k_{q} \boldsymbol{I}_{3 \times 3} & \mathbf{0}_{3,1} & \mathbf{0}_{3 \times 3} \\
0 & \mathbf{0}_{1,3} & 0 & \mathbf{0}_{1,3} \\
\mathbf{0}_{3,1} & \mathbf{0}_{3,3} & \mathbf{0}_{1 \times 3} & k_{p} \boldsymbol{I}_{3 \times 3}
\end{array}\right]
$$

with $k_{q}>0$ and $k_{p}>0$. The term (13) ensures that if $\hat{z} \rightarrow 0$ then $\hat{\zeta} \rightarrow 0$. Consider the Lyapunov function $V$ : $\mathbb{R} \times \mathbb{R}^{8} \times \mathbb{R}^{8} \rightarrow \mathbb{R}$ defined as

$$
V=\left(\hat{q}_{e}-\hat{q}_{I}\right)^{\top}\left(\hat{q}_{e}-\hat{q}_{I}\right)+\frac{1}{2} \hat{z}^{\top} \hat{K} \hat{M} \hat{z} .
$$

where we have used the fact that $\hat{\mathbb{H}}_{u}$ and $\hat{\mathbb{H}}_{v}$ are isomorphic to $\mathbb{R}^{8}$ where $\hat{K}$ is anti-diagonal matrix defined as

$$
\hat{K}=\left[\begin{array}{cccc}
0 & \mathbf{0}_{1,3} & 1 & \mathbf{0}_{1,3} \\
\mathbf{0}_{3,1} & \mathbf{0}_{3 x 3} & \mathbf{0}_{3,1} & \boldsymbol{I}_{3 \times 3} \\
1 & \mathbf{0}_{1,3} & 0 & \mathbf{0}_{1,3} \\
\mathbf{0}_{3,1} & \boldsymbol{I}_{3 \times 3} & \mathbf{0}_{1 \times 3} & \mathbf{0}_{3,3}
\end{array}\right] .
$$

such that $\hat{K} \hat{M}$ is symmetric and positive definite. It is a valid Lyapunov function since $V>0$ when $\left(\hat{q}_{e}, \hat{z}\right) \in \hat{\mathbb{H}}_{u} \times \hat{\mathbb{H}}_{v} \backslash$ $\left\{\hat{q}_{I}, 0\right\}$ and $V=0$ when $\left(\hat{q}_{e}, \hat{z}\right)=\left(\hat{q}_{I}, 0\right)$. The derivative of (15) is

$$
\begin{aligned}
\dot{V}= & \hat{\zeta}^{\top}\left(\hat{z}-\hat{K}_{1} \hat{\zeta}\right) \\
& +\hat{z}^{\top} \hat{K}\left(\hat{f}_{u}^{b}-\hat{f}_{d}^{b}-\hat{f}_{G}^{b}-\hat{\omega}^{b} \times \hat{M} \hat{\omega}^{b}-\hat{M}_{\dot{\hat{\omega}}_{c}^{b}}^{b} .\right.
\end{aligned}
$$

Inserting (10) in (17) yields

$$
\dot{V}=-\hat{K}_{1} \hat{\zeta}^{\top} \hat{\zeta}-\hat{K}_{2} \hat{z}^{\top} \hat{z} \leq 0
$$

which implies that $V$ is a decreasing function and converges to the set $S=\left\{\left(\hat{q}_{e}, \hat{z}\right) \in \hat{\mathbb{H}}_{u} \times \hat{\mathbb{H}}_{v} \mid \dot{V}=0\right\}$. Since $\dot{V}=$ 0 in $S$ it implies that $\hat{\zeta}=0$ and therefore $\boldsymbol{q}_{e, v}=0$ and $\boldsymbol{p}_{e}^{b}=$ 
0 . It then follows from (8) that $\hat{q}_{e}=\hat{q}_{I}$. Therefore $\left(\hat{q}_{e}, \hat{z}\right) \rightarrow$ $\left(\hat{q}_{I}, 0\right)$ such that from Assumption 3 the equilibrium point $\left(\hat{q}_{I}, 0\right)$ is uniformly asymptotically stable. The term $\dot{\hat{\omega}}_{c}^{b}$ in (10) can be expanded as

$$
\dot{\hat{\omega}}_{c}^{b}=\dot{\hat{\omega}}_{d}^{b}+\hat{\omega}_{d}^{b} \times \hat{\omega}_{e}^{b}-\hat{K}_{1} \dot{\hat{\zeta}}
$$

where

$$
\dot{\hat{\zeta}}=\frac{1}{2}\left(q_{0} \boldsymbol{\omega}_{e}^{b}+\boldsymbol{q}_{v} \times \boldsymbol{\omega}_{e}^{b}+\epsilon\left(\boldsymbol{v}_{e}^{b}-\boldsymbol{\omega}_{i, b}^{b} \times \boldsymbol{p}_{e}^{b}\right)\right) .
$$

Remark Since the primary part of a dual-quaternion is a unit-quaternion there are two equilibrium points for $\hat{q}_{e}$. This ambiguity will cause unwinding which is a well known problem and several solutions to this issue have been proposed for instance [21] and references therein.

\section{Adaptive backstepping}

In this section an adaptive integrator backstepping control law is derived that relaxes Assumption 2 and moreover does not assume that the disturbance $\hat{f}_{d}^{b}$ is known. To enable adaptive control design the dynamic equation (7) is rewritten as

$$
\hat{M} \dot{\hat{\omega}}^{b}=\hat{f}_{u}^{b}-\hat{f}_{d}^{b}-\left(\mathrm{L}\left(\hat{g}^{b}\right)+\hat{\mathbf{S}}\left(\hat{\omega}^{b}\right) \mathrm{L}\left(\hat{\omega}^{b}\right)\right) \boldsymbol{\theta}
$$

where

$$
\hat{\mathbf{S}}\left(\hat{\omega}^{b}\right)=\left[\begin{array}{cccc}
0 & \mathbf{0}_{1 \times 3} & 0 & \mathbf{0}_{1 \times 3} \\
\mathbf{0}_{3 \times 1} & S\left(\boldsymbol{\omega}_{i, b}^{b}\right) & \mathbf{0}_{3 \times 1} & \mathbf{0}_{3 \times 3} \\
0 & \mathbf{0}_{1 \times 3} & 0 & \mathbf{0}_{1 \times 3} \\
\mathbf{0}_{3 \times 1} & S\left(\boldsymbol{v}^{b}\right) & \mathbf{0}_{3 \times 1} & S\left(\boldsymbol{\omega}_{i, b}^{b}\right)
\end{array}\right]
$$

and for a dual-vector $\hat{\alpha}=\boldsymbol{\alpha}_{p}+\epsilon \boldsymbol{\alpha}_{d}, \boldsymbol{L}(\hat{\alpha})$ is defined as

$$
\mathrm{L}(\hat{\alpha})=\left[\begin{array}{cccc}
0 & 0 & \mathbf{0}_{1 \times 3} & \mathbf{0}_{1 \times 3} \\
\mathbf{0}_{3 \times 1} & \boldsymbol{\alpha}_{d} & \mathbf{0}_{1 \times 3} & \mathbf{0}_{3 \times 3} \\
0 & 0 & \mathbf{0}_{1 \times 3} & \mathbf{0}_{1 \times 3} \\
\mathbf{0}_{3 \times 1} & \mathbf{0}_{3 \times 1} & \operatorname{diag}\left(\boldsymbol{\alpha}_{p}\right) & D\left(\boldsymbol{\alpha}_{p}\right)
\end{array}\right] .
$$

The unknown parameter vector $\boldsymbol{\theta}$ is defined as

$$
\boldsymbol{\theta}=\left[\begin{array}{llllllll}
0 & m & J_{x} & J_{y} & J_{z} & J_{x y} & J_{x z} & J_{y z}
\end{array}\right]^{\top} .
$$

The control law (10) can then be rewritten as

$$
\begin{aligned}
\hat{f}_{u}^{b}= & \hat{f}_{d}^{b}+\left(\mathrm{L}\left(\hat{g}^{b}\right)+\hat{\mathrm{S}}\left(\hat{\omega}^{b}\right) \mathrm{L}\left(\omega^{b}\right)+\mathrm{L}\left(\dot{\hat{\omega}}_{c}^{b}\right)\right) \boldsymbol{\theta} \\
& -\hat{K}\left(\hat{K}_{2} \hat{z}+\hat{\zeta}\right) .
\end{aligned}
$$

Since $\boldsymbol{\theta}$ and $\hat{f}_{d}^{b}$ are unknown we replace them by their estimates $\overline{\boldsymbol{\theta}}$ and $\overline{\hat{f}}_{d}^{b}$, respectively, to obtain the certainty equivalence controller

$$
\begin{aligned}
\hat{f}_{u}^{b}= & \overline{\hat{f}}_{d}^{b}+\left(\mathrm{L}\left(\hat{g}^{b}\right)+\hat{\mathrm{S}}\left(\hat{\omega}^{b}\right) \mathrm{L}\left(\omega^{b}\right)+\mathrm{L}\left(\dot{\hat{\omega}}_{c}^{b}\right)\right) \overline{\boldsymbol{\theta}} \\
& -\hat{K}\left(\hat{K}_{2} \hat{z}+\hat{\zeta}\right)
\end{aligned}
$$

Define the unknown parameter errors as

$$
\tilde{\boldsymbol{\theta}}=\overline{\boldsymbol{\theta}}-\boldsymbol{\theta}, \quad \tilde{\hat{f}}_{d}^{b}=\overline{\hat{f}}_{d}^{b}-\hat{f}_{d}^{b}
$$

and augment the Lyapunov candidate function (15) to

$$
\begin{aligned}
V\left(\hat{q}_{e}, \hat{z}\right)= & \left(\hat{q}_{e}-\hat{q}_{I}\right)^{\top}\left(\hat{q}_{e}-\hat{q}_{I}\right)+\frac{1}{2} \hat{z}^{\top} \hat{K} \hat{M} \hat{z} \\
& +\frac{1}{2} \tilde{\boldsymbol{\theta}}^{\top} \boldsymbol{\Gamma}_{\theta}^{-1} \tilde{\boldsymbol{\theta}}+\frac{1}{2} \tilde{\hat{f}}_{d}^{b \top} \boldsymbol{\Gamma}_{d}^{-1} \tilde{\hat{f}}_{d}^{b}
\end{aligned}
$$

where $\boldsymbol{\Gamma}_{\theta}$ and $\boldsymbol{\Gamma}_{d}$ are positive definite and symmetric adaptation gain matrices. Let $\lambda_{\max }=\max \left(\|\hat{M}\|,\left\|\boldsymbol{\Gamma}_{\theta}^{-1}\right\|,\left\|\boldsymbol{\Gamma}_{d}^{-1}\right\|\right)$ and

$$
\chi=\left[\begin{array}{lllll}
\boldsymbol{q}_{e, p}-\boldsymbol{q}_{I} & \boldsymbol{q}_{e, d} & \boldsymbol{z}_{p} & \boldsymbol{z}_{d} & \boldsymbol{\theta}
\end{array}\right]^{\top} \in \mathbb{R}^{24}
$$

such that $\frac{1}{2\left(1+\left|\lambda_{\max }\right|\right)}\|\chi\|^{2} \leq V(\chi) \leq 2\left(1+\left|\lambda_{\max }\right|\right)\|\chi\|^{2}$. The derivative then becomes

$$
\begin{aligned}
\dot{V}= & -\hat{K}_{1} \hat{\zeta}^{\top} \hat{\zeta}+\hat{\zeta}^{\top} \hat{z} \\
& +\hat{z} \hat{K}\left(\hat{f}_{u}^{b}-\hat{f}_{d}^{b}-\left(\mathrm{L}\left(\hat{g}^{b}\right)+\hat{\mathrm{S}}\left(\hat{\omega}^{b}\right) \mathrm{L}\left(\hat{\omega}^{b}\right)\right.\right. \\
& \left.\left.+\mathrm{L}\left(\dot{\hat{\omega}}_{c}^{b}\right)\right) \boldsymbol{\theta}\right)+\dot{\overline{\boldsymbol{\theta}}}^{\top} \boldsymbol{\Gamma}_{\theta}^{-1} \tilde{\boldsymbol{\theta}}+\dot{\overline{\hat{f}}}_{d}^{b \top} \boldsymbol{\Gamma}_{d}^{-1} \tilde{\hat{f}}_{d}^{b}
\end{aligned}
$$

Inserting the certainty equivalence controller (25) into (29) results in

$$
\begin{aligned}
\dot{V}= & -\hat{K}_{1} \hat{\zeta}^{\top} \hat{\zeta}-\hat{K}_{2} \hat{z}^{\top} \hat{z} \\
& +\left(\hat{z}^{\top} \hat{K}\left(\mathrm{~L}\left(\hat{g}^{b}\right)+\hat{\mathbf{S}}\left(\hat{\omega}^{b}\right) \mathbf{L}\left(\hat{\omega}^{b}\right)+\mathrm{L}\left(\dot{\hat{\omega}}_{c}^{b}\right)\right)\right. \\
& \left.+\dot{\overline{\boldsymbol{\theta}}}^{\top} \boldsymbol{\Gamma}_{\theta}^{-1}\right) \tilde{\boldsymbol{\theta}}+\left(\hat{z}^{\top} \hat{K}+\dot{\hat{\hat{f}}}_{d}^{\top} \boldsymbol{\Gamma}_{d}^{-1}\right) \tilde{\hat{f}}_{d}^{b} .
\end{aligned}
$$

By choosing the update laws

$$
\dot{\overline{\boldsymbol{\theta}}}=-\left(\hat{z}^{\top} \hat{K}\left(\mathbf{L}\left(\hat{g}^{b}\right)+\hat{\mathbf{S}}\left(\hat{\omega}^{b}\right) \mathbf{L}\left(\hat{\omega}^{b}\right)+\mathbf{L}\left(\dot{\hat{\omega}}_{c}^{b}\right)\right) \boldsymbol{\Gamma}_{\theta}\right)^{\top}
$$

and

$$
\dot{\overline{\hat{f}}}_{d}^{b}=-\left(\hat{z}^{\top} \hat{K} \boldsymbol{\Gamma}_{d}\right)^{\top}
$$

the derivative becomes

$$
\dot{V}=-\hat{K}_{1} \hat{\zeta}^{\top} \hat{\zeta}-\hat{K}_{2} \hat{z}^{\top} \hat{z} \leq 0
$$

which implies that $\hat{q}_{e}$ and $\hat{z}$ are bounded. Let $k_{3}=$ $\min \left(k_{q}, k_{p}, k_{\omega}, k_{v}\right)$ and define $W=\frac{k_{3}}{2}\|\hat{\zeta}\|^{2}+\frac{k_{3}}{2}\|\hat{z}\|^{2}$ then $W \leq-\dot{V}$ such that

$$
\lim _{t \rightarrow \infty} \int_{t_{0}}^{t} W(\chi(\tau)) \mathrm{d} \tau \leq V_{t_{0}}-V_{\infty}
$$

which exists and is finite since $V$ is positive definite, decreasing and bounded from below. Since $W$ is positive definite and continuous it follows from Assumption 3 that the equilibrium point $\left(\hat{q}_{I}, 0\right)$ is uniformly asymptotically stable.

Remark The term $\dot{\hat{\omega}}_{c}^{b}$ is defined in (18) and (19), which requires the knowledge of $\dot{\hat{\omega}}_{d}^{b}$ which is assumed to be known from Assumption 3.

\section{Simulation}

In this section we discuss some numerical examples using the daptive controller derived in Section IV-D. Two simulation cases are presented. In the first case a desired trajectory is tracked with uknown mass and inertia parameters in addition to an unknown constant or slowly time-varying disturbance dual-force. In the second case it is demonstrated that the estimated mass and inertia parameters converge to the true values when a desired trajectory is defined in accordance with the conditions outlined in [17]. 


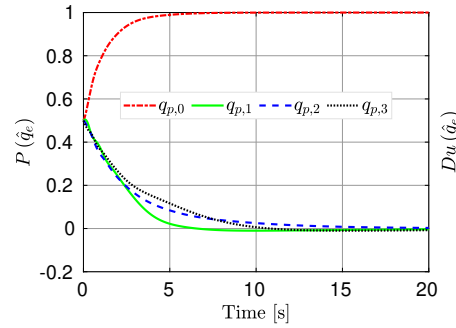

(a) Primary part.

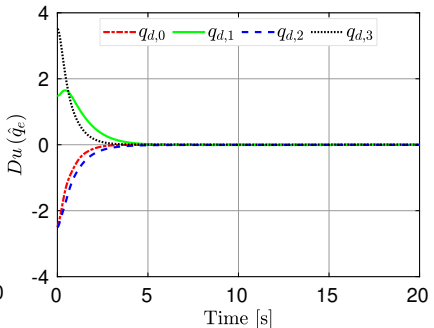

(b) Dual part.
Fig. 1: The dual-quaternion error $\hat{q}_{e}$

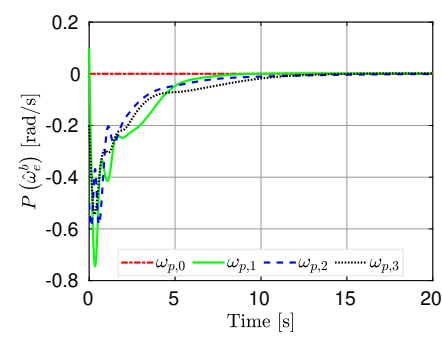

(a) Primary part.

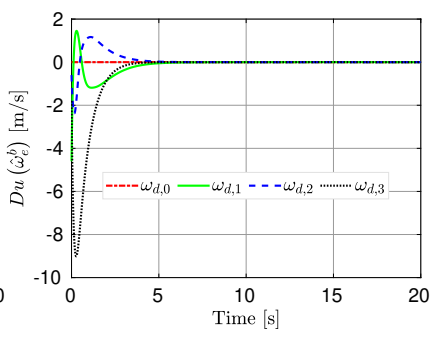

(b) Dual part.
Fig. 2: The dual-velocity error $\hat{q}_{e}$

\section{A. Case I}

The desired trajectory for the rigid-body to follow is defined as [20]

$$
\begin{aligned}
\boldsymbol{p}_{d}^{i} & =\left[\begin{array}{llll}
0 & r \sin \left(\omega_{0} t\right) & r \cos \left(\omega_{0} t\right) & 10
\end{array}\right] \\
\dot{\boldsymbol{p}}_{d}^{i} & =\left[\begin{array}{llll}
0 & \omega_{0} r \cos \left(\omega_{0} t\right) & -\omega_{0} r \sin \left(\omega_{0} t\right) & 0
\end{array}\right] \\
\ddot{\boldsymbol{p}}_{d}^{i} & =\left[\begin{array}{llll}
0 & -\omega_{0}^{2} r \sin \left(\omega_{0} t\right) & -\omega_{0}^{2} r \cos \left(\omega_{0} t\right) & 0
\end{array}\right]
\end{aligned}
$$

where $\omega_{0}=0.1$ and $r=10$ which describes a circle with radius 10 meters at an altitude of 10 meters. The desired trajectory for the orientation is

$$
\begin{aligned}
\boldsymbol{q}_{i, d}\left(t_{0}\right) & =\boldsymbol{q}_{I}, \boldsymbol{\omega}_{i, d}^{d}\left(t_{0}\right)=\left[\begin{array}{llll}
0 & 0.2 & -0.1 & 0.5
\end{array}\right]^{\top} \\
\dot{\boldsymbol{\omega}}_{i, d}^{d} & =\left[\begin{array}{llll}
0 & 0 & 0 & 0
\end{array}\right]^{\top} .
\end{aligned}
$$

The desired dual-quaternion and dual-velocity is constructed based on (4) and (5) in Section III-A. The initial conditions for the rigid-body is $\hat{q}\left(t_{0}\right)=$ $\left[\begin{array}{llll}0.5 & 0.5 & 0.5 & 0.5\end{array}\right]^{\top}+\epsilon\left[\begin{array}{llll}-7.5 & 1.5 & 2.5 & 3.5\end{array}\right]^{\top}$ and $\hat{\omega}^{b}\left(t_{0}\right)=\mathbf{0}+\epsilon \mathbf{0}$. The controller gains are set

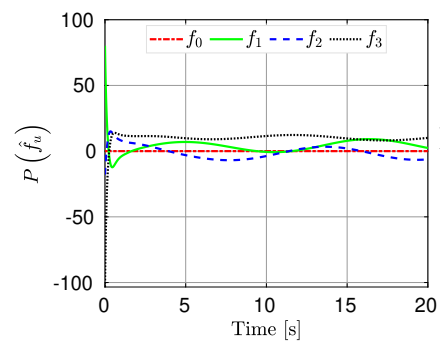

(a) Primary part.

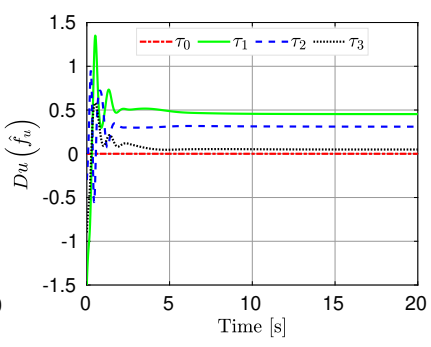

(b) Dual part.
Fig. 3: The dual control force. to $\hat{K}_{1}=\operatorname{diag}\left(\left[\begin{array}{llllllll}0 & 0.8 & 0.8 & 0.8 & 0 & 2 & 2 & 2\end{array}\right]^{\top}\right)$ and $\hat{K}_{2}=\operatorname{diag}\left(\left[\begin{array}{llllllll}0 & 2 & 2 & 2 & 0 & 12 & 12 & 12\end{array}\right]^{\top}\right)$ while $\hat{K}$ is defined as in (16). The initial values for the estimated mass and inertia parameters are set to $\bar{\theta}\left(t_{0}\right)=\left[\begin{array}{llllllll}0 & 0 & 0 & 0 & 0 & 0 & 0 & 0\end{array}\right]^{\top}$ while the initial value for the estimated disturbance dual-force is $\hat{f}_{d}^{b}\left(t_{0}\right)=\mathbf{0}+\epsilon \mathbf{0}$. The disturbance dual-force in (7) is set to $\hat{f}_{d}^{b}=\frac{1}{2}\left[\begin{array}{llll}0 & 0.8147 & 0.9058 & 0.1270\end{array}\right]^{\top}+$ $\epsilon \frac{1}{2}\left[\begin{array}{llll}0 & 0.9134 & 0.6324 & 0.0975\end{array}\right]^{\top}$ which has a magnitude of $\left\|\hat{f}_{d}^{b}\right\|=0.8283$. The adaption gain matrices are set to

$$
\begin{aligned}
& \boldsymbol{\Gamma}_{\theta}=\operatorname{diag}\left(100 \cdot\left[\begin{array}{llllllll}
0 & 0.001 & 1 & 1 & 1 & 1 & 1 & 1
\end{array}\right]^{\top}\right) \\
& \boldsymbol{\Gamma}_{d}=\operatorname{diag}\left(0.5 \cdot\left[\begin{array}{llllllll}
0 & 1 & 1 & 1 & 0 & 1 & 1 & 1
\end{array}\right]^{\top}\right) .
\end{aligned}
$$

It can be seen from Figure 1 and Figure 2, which shows the error dual-quaternion and error dual-velocities, that the trajectory of the rigid-body converges to the desired trajectory. From Figure 1a it is seen that $\boldsymbol{q}_{e} \rightarrow \boldsymbol{q}_{I}$, where $\boldsymbol{q}_{e}$ is the primary part of the error dual-quaternion, which implies that $\boldsymbol{q}_{i, b} \rightarrow \boldsymbol{q}_{i, d}$. In Figure $1 \mathrm{~b}$ the dual part of $\hat{q}$ goes toward zero and since it is defined as $\epsilon \frac{1}{2} \boldsymbol{p}_{e}^{i} \otimes \boldsymbol{q}_{e}$ it implies that $\boldsymbol{p}^{i} \rightarrow \boldsymbol{p}_{d}^{i}$. In Figure $2 \mathrm{~b}$ it can be seen that the dual velocity error goes to zero which implies that $\hat{\omega}^{b} \rightarrow \hat{\omega}_{d}^{d}$. There are some oscillations in the primary part of the dual velocity which can also be observed in the dual part of the dual control force in Figure 3. These oscillations are a result of the disturbance dual force, because it takes some time for the adaptive control law estimate the disturbance force such that good compensation is achieved. After about 100 seconds $\left\|\hat{q}_{e}-\hat{q}_{I}\right\|$ is less than 0.0004 , while $\left\|\hat{\omega}^{b}\right\|$ is less than 0.0002 .

\section{B. Case II}

Let the initial conditions be defined as

$$
\begin{aligned}
\boldsymbol{q}_{i, d}\left(t_{0}\right) & =\boldsymbol{q}_{I}, \quad \boldsymbol{\omega}_{i, d}^{d}\left(t_{0}\right)=\left[\begin{array}{lll}
0 & 0 & 0
\end{array}\right]^{\top} \\
\dot{\boldsymbol{\omega}}_{i, d}^{d}= & =\left[\begin{array}{lll}
c_{1} \cos c_{2} t & c_{3} \cos c_{4} t+\frac{\pi}{9} & c_{5} \pi \cos c_{6} t+\frac{2 \pi}{9}
\end{array}\right]^{\top} \\
\boldsymbol{p}^{i}\left(t_{0}\right)= & \boldsymbol{q}_{I}, \quad \boldsymbol{v}^{i}\left(t_{0}\right)=\left[\begin{array}{lll}
0 & 0 & 0
\end{array}\right]^{\top} \\
\dot{\boldsymbol{v}}^{d}= & {\left[\begin{array}{lll}
c_{1} \cos c_{2} t & c_{3} \cos c_{4} t+\frac{\pi}{9} & c_{5} \pi \cos c_{6} t+\frac{2 \pi}{9}
\end{array}\right]^{\top} } \\
& +\boldsymbol{\omega}_{i, d}^{d} \times \boldsymbol{v}^{d}
\end{aligned}
$$

where $c_{1}=-0.02 \pi, c_{2}=0.2 \pi, c_{3}=-0.02 \pi, c_{4}=0.1 \pi$, $c_{5}=-0.02 \pi$ and $c_{6}=0.0667 \pi$. The mass and inertia parameters are $m=1, J_{x x}=1, J_{y y}=0.63, J_{z z}=0.86$ and $J_{x y}=J_{x z}=J_{y z}=0$. The desired dual-quaternion and dual-velocity is constructed based on (4) and (5) in Section III-A. The initial conditions for the rigid-body is

$$
\begin{aligned}
& \hat{q}\left(t_{0}\right)=\left[\begin{array}{c}
0.33 \\
0.46 \\
0.19 \\
0.8
\end{array}\right]+\epsilon\left[\begin{array}{c}
-7.27 \\
-1.38 \\
3.35 \\
3.01
\end{array}\right], \hat{\omega}^{b}\left(t_{0}\right)=\left[\begin{array}{c}
0 \\
0.1 \\
0.1 \\
0.1
\end{array}\right]+\epsilon\left[\begin{array}{c}
0 \\
0.1 \\
0.1 \\
0.1
\end{array}\right] . \\
& \text { The controller gains set to } \hat{K}_{1}= \\
& \operatorname{diag}\left(\left[\begin{array}{llllllll}
0 & 0.5 & 0.5 & 0.5 & 0 & 0.1 & 0.1 & 0.1
\end{array}\right]^{\top}\right) \quad \text { and } \\
& \hat{K}_{2}=\operatorname{diag}\left(\left[\begin{array}{llllllll}
0 & 8 & 8 & 8 & 0 & 8 & 8 & 8
\end{array}\right]^{\top}\right) \quad \text { while }
\end{aligned}
$$



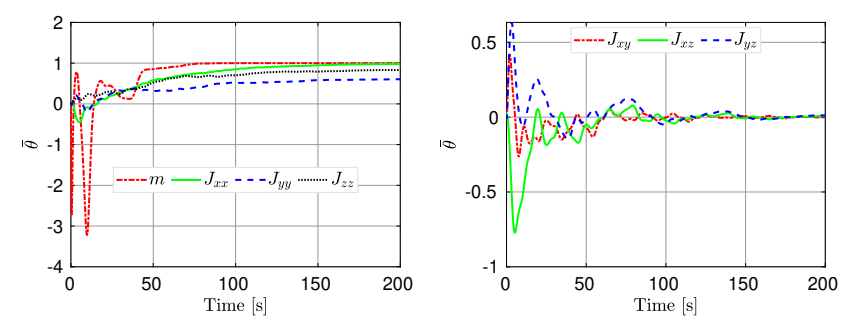

(a) At 200 s, $m \approx 1, J_{x x} \approx 1$,(b) At 200 s, $J_{x y} \approx 0, J_{x z} \approx 0.0$ $J_{y y} \approx 0.63$ and $J_{z z} \approx 0.85$. and $J_{y x} \approx 0.0$.

Fig. 4: Estimated mass and inertia parameters.

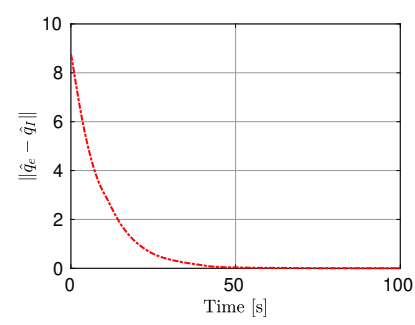

(a) $\left\|\hat{q}_{e}-\hat{q}_{I}\right\|$.

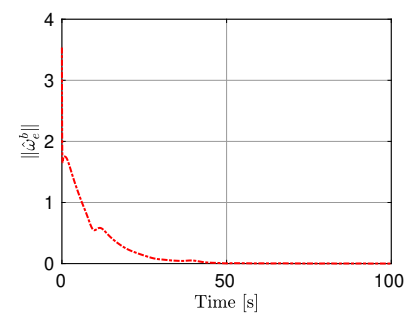

(b) $\left\|\hat{\omega}_{e}^{b}\right\|$.
Fig. 5: Errors

$\hat{K}$ is defined as in (16). The initial values for the estimated mass and inertia parameters are set to $\bar{\theta}\left(t_{0}\right)=\left[\begin{array}{llllllll}0 & 0 & 0 & 0 & 0 & 0 & 0 & 0\end{array}\right]^{\top}$ while the disturbance dual-force and gravity force is set to $\hat{f}_{d}^{b}\left(t_{0}\right)=\hat{f}_{g}^{b}=\mathbf{0}+\epsilon \mathbf{0}$. The adaption gain matrices are set to

$$
\begin{aligned}
\boldsymbol{\Gamma}_{\theta} & =\operatorname{diag}\left(100 \cdot\left[\begin{array}{llllllll}
0 & 0.1 & 1 & 1 & 1 & 1 & 1 & 1
\end{array}\right]^{\top}\right) \\
\boldsymbol{\Gamma}_{d} & =\operatorname{diag}\left(0.5 \cdot\left[\begin{array}{lllllllll}
0 & 1 & 1 & 1 & 0 & 1 & 1 & 1
\end{array}\right]^{\top}\right) .
\end{aligned}
$$

As can be seen from Figure 4 the estimated mass and inertia matrix converge to the true mass and inertia matrix. The proof is found in [17] and can be adapted to the case of the proposed controller in this paper. The error is seen in Figure 5 and it can be concluded from the figure that $\left(\hat{q}, \hat{\omega}^{b}\right) \rightarrow\left(\hat{q}_{d}, \hat{\omega}_{d}^{d}\right)$. It is also seen that that the it takes longer for the adaptive controller to estimate the mass and inertia parameters than it takes for the system to track the desired trajectory.

\section{CONCLUSIONS}

In this paper the trajectory tracking problem for a fully actuated rigid-body with unknown mass and inertia properties and unknown disturbance forces was solved in a dual-quaternion framework using an adaptive backstepping controller. The certainty equivalence adaptive backstepping controller was presented and proved to make the equilibrium points of the error system uniformely asymptotically stable. A numerical example was provided through a simulation which demonstrates the proposed controller. Additionally it was shown by a simulation example that by choosing a proper desired trajectory the estimated mass and inertia parameters will converge to the true mass and inertia parameters. Future work will focus on extending the adaptive backstepping controller to an ISS-controller with a leastsquares identifier to estimate the true values of the mass and inertia parameters for wider class of desired trajectories.

\section{REFERENCES}

[1] E. Study, "Von den Bewegungen und Umlegungen," Mathematische Annalen, vol. 39, no. 4, pp. $441-565,1892$.

[2] W. K. Clifford, "Preliminary sketch of biquaternions," In Proc. of the London Mathematical Society, vol. s1-4, no. 1, pp. 381-395, 1871.

[3] B. Ravani and B. Roth, "Mappings of spatial kinematics," Journal of Mechanisms, Transmissions, and Automation in Design, vol. 106, no. 3, pp. 341 - 347, 1984.

[4] V. Brodsky and M. Shoham, "Dual numbers representation of rigid body dynamics," Mechanisms and Machine Theory, vol. 34, no. 5, pp. $693-718,1999$.

[5] J.-Y. Wang, H.-Z. Liang, Z.-W. Sun, S.-N. Wu, and S.-J. Zhang, "Relative motion coupled control based on dual quaternion," Aerospace Science and Technology, vol. 25, no. 1, pp. $102-113,2013$.

[6] J. R. Dooley and J. M. McCarthy, "Spatial rigid body dynamics using dual quaternion components," in In Proc. of IEEE International Conference on Robotics and Automation, vol. 1, pp. 90-95, 1991.

[7] Y. Wu, X. Hu, D. Hu, T. Li, and J. Lian, "Strapdown inertial navigation system algorithms based on dual quaternions," IEEE Transactions on Aerospace and Electronic Systems, vol. 41, no. 1, pp. 110-132, 2005.

[8] X. Wang and C. Yu, "Unit dual quaternion-based feedback linearization tracking problem for attitude and position dynamics," Systems \& Control Letters, vol. 62, no. 3, pp. 225 - 233, 2013.

[9] N. Filipe and P. Tsiotras, "Simultaneous position and attitude control without linear and angular velocity feedback using dual quaternions,' in In Proc. of American Control Conference, 2013.

[10] U. Lee and M. Mesbahi, "Dual quaternions, rigid body mechanics, and powered-descent guidance," in In Proc. of IEEE Conference on Decision and Control, pp. 3386-3391, 2012.

[11] T.-A. Johansen, T. S. Andersen, and R. Kristiansen, "PD+ Based Trajectory Tracking of the Underactuated Quadrotor Platform using Dual Quaternions," in In Proc. of American Control Conference, pp. 1342-1348, 2019.

[12] H. Gui and G. Vukovich, "Finite-time output-feedback position and attitude tracking of a rigid body," Automatica, vol. 74, pp. 270 - 278, 2016.

[13] F. Zhang and G. Duan, "Robust Integrated Translation and Rotation Finite-Time Maneuver of a Rigid Spacecraft Based on Dual Quaternion," In Proc. of AIAA Guidance, Navigation, and Control Conference, 2011.

[14] H. Gui and G. Vukovich, "Dual-quaternion-based adaptive motion tracking of spacecraft with reduced control effort," Nonlinear Dynamics, vol. 83, no. 1, pp. 597-614, 2016.

[15] N. Filipe and P. Tsiotras, "Adaptive Position and Attitude-Tracking Controller for Satellite Proximity Operations Using Dual Quaternions," Journal of Guidance, Control, and Dynamics, vol. 38, no. 4, pp. 566$577,2015$.

[16] A. Valverde and P. Tsiotras, "Spacecraft Trajectory Tracking with Identification of Mass Properties Using Dual Quaternions," In Proc. of AIAA Guidance, Navigation, and Control Conference, 2018.

[17] N. Filipe and P. Tsiotras, "Adaptive Model-Independent Tracking of Rigid Body Position and Attitude Motion with Mass and Inertia Matrix Indentification using Dual Quaternions," AIAA Guidance, Navigation, and Control Conference, 2013.

[18] U. Lee and M. Mesbahi, "Dual Quaternion based Spacecraft Rendezvous with Rotational and Translational Field of View Constraints,' in In Proc. of AIAA/AAS Astrodynamics Specialist Conference, 2014.

[19] V. Brodsky and M. Shoham, "The Dual Inertia Operator and Its Application to Robot Dynamics," Journal of Mechanical Design, vol. 116, no. 4, pp. 1089-1095, 1994.

[20] T. Andersen, T. Johansen, and R. Kristiansen, "Dual-Quaternion Backstepping Control for a Fully-Actuated Rigid-Body," in In Proc. of American Control Conference, pp. 5653-5658, 2018.

[21] R. Schlanbusch, R. Kristiansen, and P. J. Nicklasson, "On choosing quaternion equilibrium point in attitude stabilization," in In Proc. of IEEE Aerospace Conference, pp. 1-6, 2010. 